\title{
Automatic detection of the unknown number point targets in FMICW radar signals
}

\author{
Lubos Rejfek $^{1, *}$, Zbysek Mosna ${ }^{2}$, Ladislav Beran ${ }^{1}$, Ondrej Fiser ${ }^{1}$, Martin Dobrovolny ${ }^{1}$ \\ ${ }^{1}$ Faculty of Electrical Engineering and Informatics, University of Pardubice, Pardubice, Czech Republic \\ ${ }^{2}$ Department of Aeronomy, Institute of Atmospheric Physics, Czech Academy of Sciences, Prague, Czech Republic
}

\section{A R T I C L E IN F O}

\section{Article history:}

Received 5 February 2017

Received in revised form

13 September 2017

Accepted 15 September 2017

Keywords:

FMICW radar

2D FFT

Signal filtration

Target detection

Target parameter estimation

\section{Introduction}

Radars are represented in many areas, for example in the air-plane navigation, in medicine areas, automatically switching of the lights and others. Radars are divided to several groups according to their principle. The first type is pulse radar, the second one is UWB (ultra-wide band), the third type is FMCW (frequency modulated continuous wave) and the next types are combinations of these radars. Systems for the automatic interpretation of the radar signals are needed for the better effectiveness of systems.

The radar discussed in our work is combination of the pulse radar and FMCW radar. Signals testing our algorithm are generated by the modified simulator described in (Mandlik and Brazda, 2015).

\section{FMICW radar}

Frequency Modulated Interrupted Continuous Wave (FMICW) radar is combination of the Frequency Modulated Continuous Wave (FMCW) radar (this type of the radar is described e.g. in (Babur et al., 2013) and pulse radar. An example of the FMICW radar is the PCDR35 radar. This radar works at the frequency $35.4 \mathrm{GHz}$. Block scheme of the PCDR35 radar is in Fig. 1 (sweeping generator), Fig. 2 (transmitter) and Fig. 3 (receiver). From the

\footnotetext{
* Corresponding Author.

Email Address: Rejfek@student.upce.cz (L. Rejfek)

https://doi.org/10.21833/ijaas.2017.011.018

2313-626X/C 2017 The Authors. Published by IASE.

This is an open access article under the CC BY-NC-ND license

(http://creativecommons.org/licenses/by-nc-nd/4.0/)
}

scheme it is possible to see that the base frequency $8.856 \mathrm{GHz}$ is multiplied by 4 for the transformation on the transmitted frequency. Switching of the transmitter and receiver is realized by the PIN diodes. Transmitter and receiver are connected to the antenna through the circulator.

Functional scheme of the FMICW radar is in Fig. 4. Frequency sweeping $(\Delta \mathrm{f})$ is realized during the whole realization $(\mathrm{T})$. Typical duration of the realization for the range $10 \mathrm{~km}$ is $133 \mu \mathrm{s}$ (66 $6 \mathrm{~s}$ is signal transmitted, $66 \mu \mathrm{s}$ is signal received and $1 \mu \mathrm{s}$ is blind zone). Output frequency ( $\delta f)$ is dependent on the target distance and speed of the frequency sweeping. Part of the reflected frequencies is lost (signals are returned when the receiver is not open). An example of the actual output signal from the radar is shown in Fig. 5. Signal is reflected from the chimney and noise is recorded in this example as well.

\section{Primary signal processing}

Signals from the radar are processed in the following two steps. In the first step the spectrum of the signal is calculated. For the spectrum analysis Fourier transform, MUSIC method, autoregressive moving average (ARMA) method and other can be used. Spectrum of the signal with one target is shown in Fig. 6. Ranges of the targets are calculated from the frequency according to the Eq. 1

$R=\delta f \cdot \frac{T C_{0}}{2 \Delta f}$

where $T$ is length of the measurement $\Delta f$ is maximum frequency sweep 
$\delta f$ is output radar frequency

$C_{0}$ is speed of the light

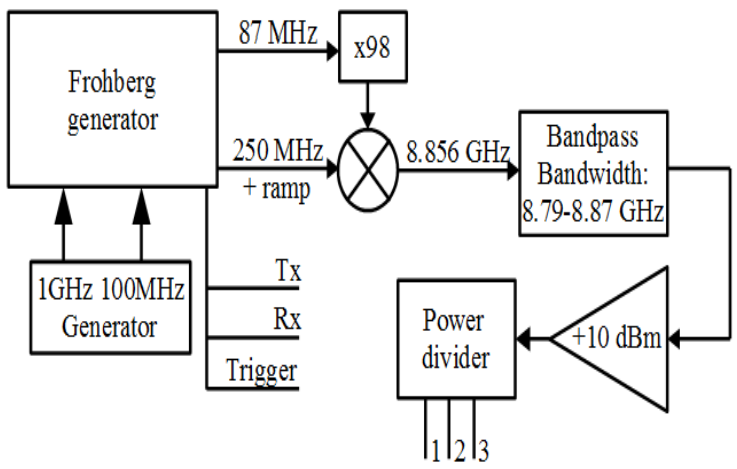

Fig. 1: Sweeping generator in FMICW radar PCDR35

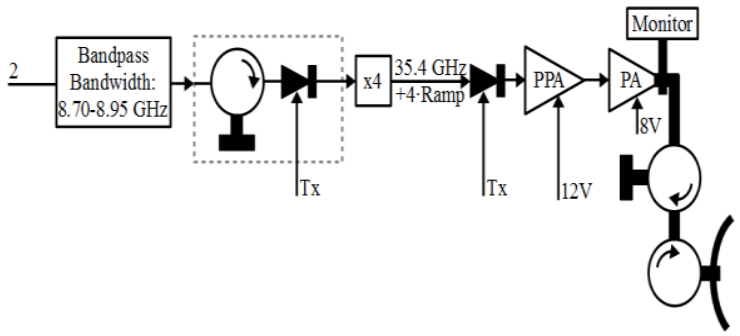

Fig. 2: Transmitter in FMICW radar PCDR35

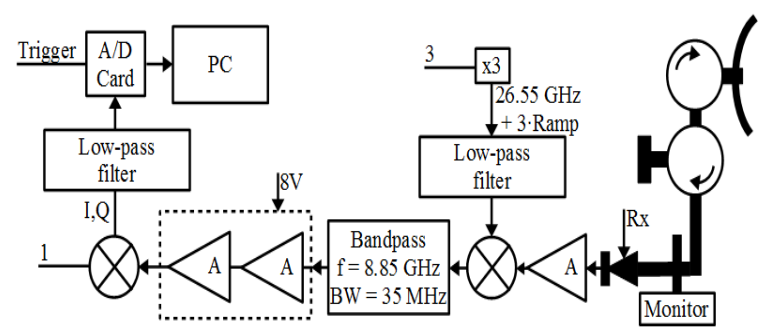

Fig. 3: Receiver in FMICW radar PCDR35

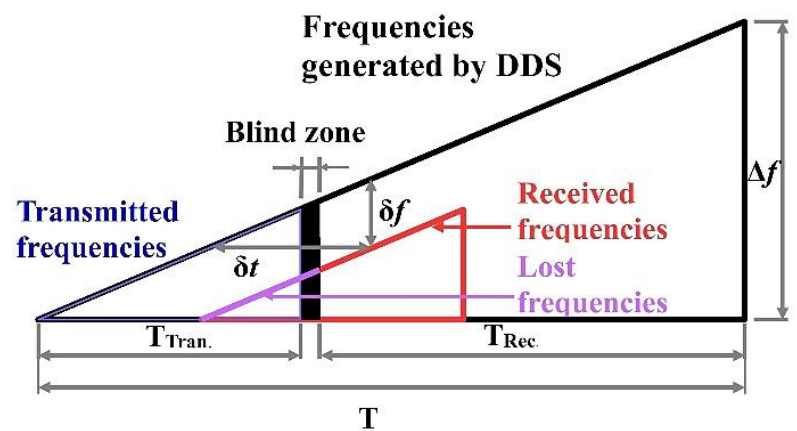

Fig. 4: Principal scheme of the FMICW radar (Rejfek et al., 2014)

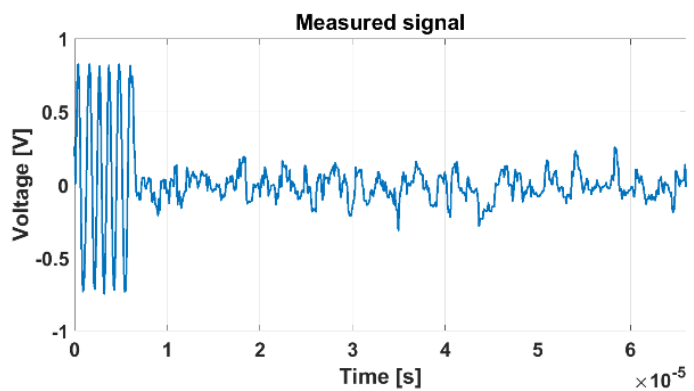

Fig. 5: An example of the received radar signal (Rejfek et al., 2016)

Spectrum of the signal must be recalculated from the ideal spectrum to real received power. This computation was described in (Rejfek et al., 2014).
Different type of the primary radar signal processing is described in ( $̌$ ezníček et al., 2016).

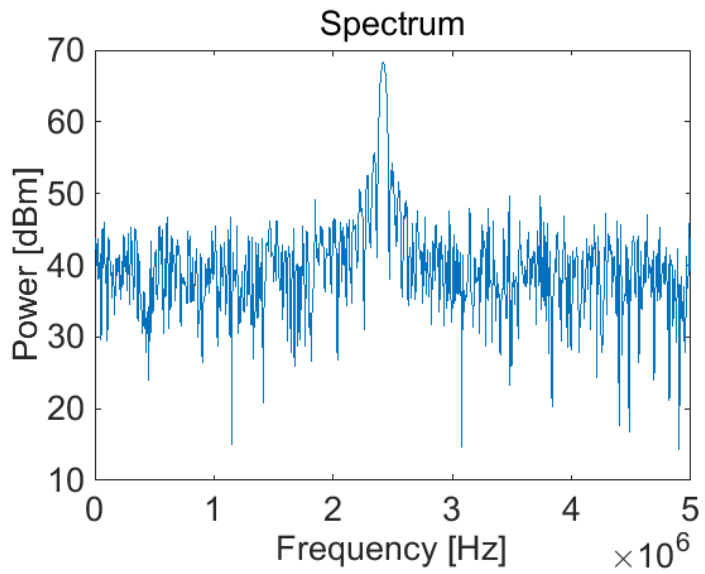

Fig. 6: Spectrum of the radar signal

The second step is calculation of the 2D spectrum. This process is described in (Mandlik and Brazda, 2015). Matrix is realized from the matrix of the measured data (the first dimension is time dimension and the second dimension is realizations). Maximal Doppler shift is calculated from the Eq. 2

$P R F_{\text {max }}=\frac{1}{2 \cdot T R}$

where $T R$ is time duration between measurements

\section{Algorithm for targets analysis}

Algorithm to estimate the number of targets and parameters of these targets is shown in diagram in Fig. 15. In this algorithm points for the validation of this algorithm are added. Input for our algorithm is output from 2D FFT (Figs. 7-14).

Our algorithm starts by the estimation of the threshold value. This value is estimated from Eq. 3 . Median is realized from the first dimension (Doppler shifts).

$T V=\operatorname{MAX}(\operatorname{MEDIAN}(S 2 D))+9$

where $\mathrm{S} 2 \mathrm{D}$ is matrix of 2D spectrum

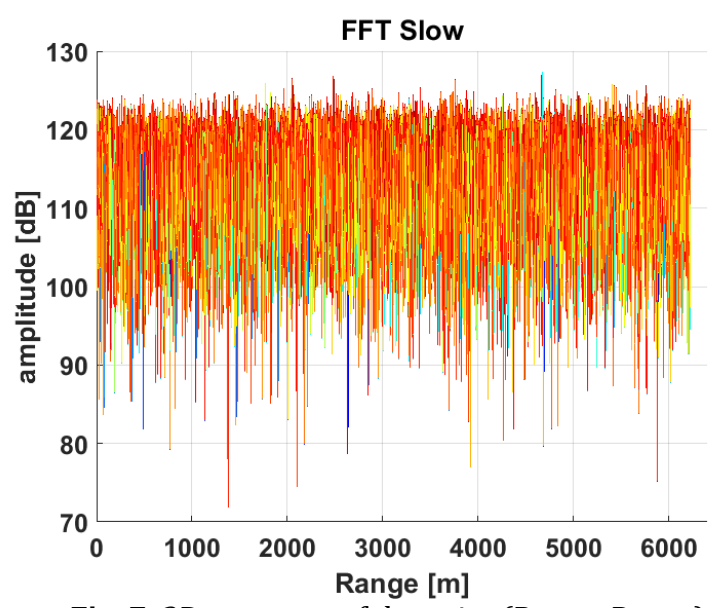

Fig. 7: 2D spectrum of the noise (Power-Range) 


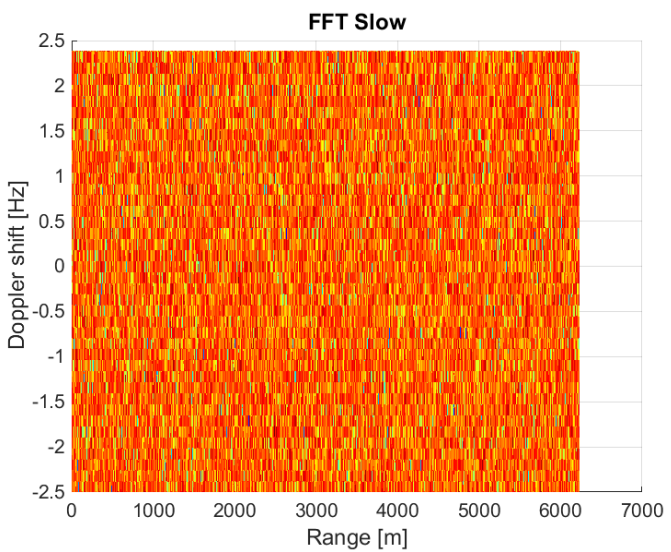

Fig. 8: 2D spectrum of the noise (Doppler shift-Range)

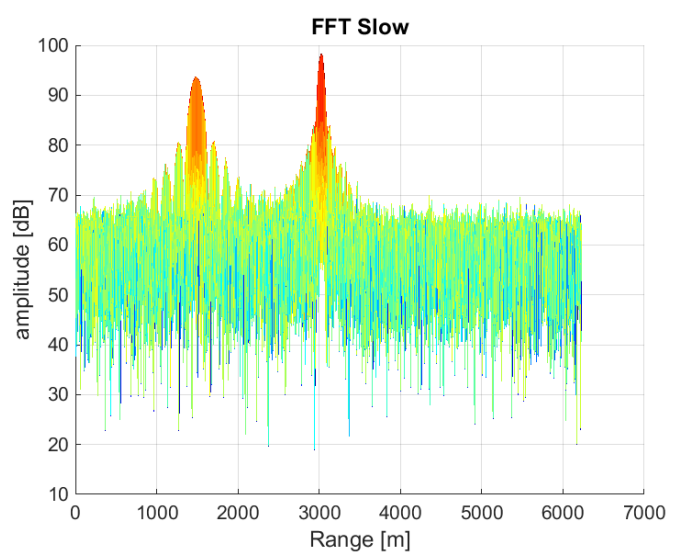

Fig. 9: 2D spectrum of the signal with two strong targets (Power-Range)

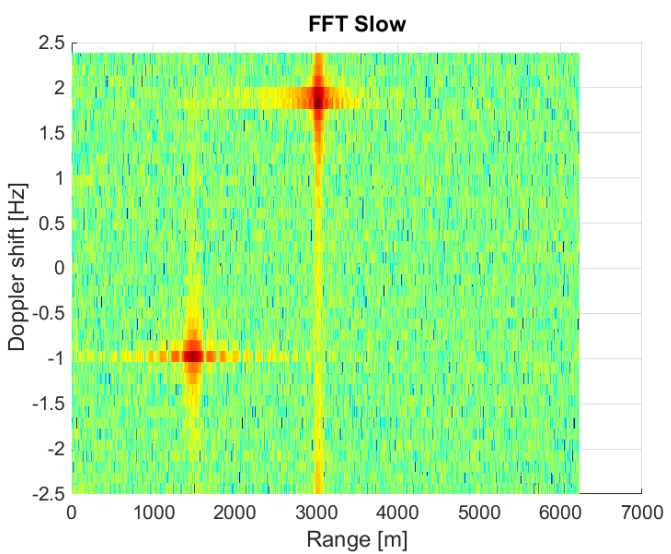

Fig. 10: 2D spectrum of the signal with two strong targets (Doppler shift-Range)

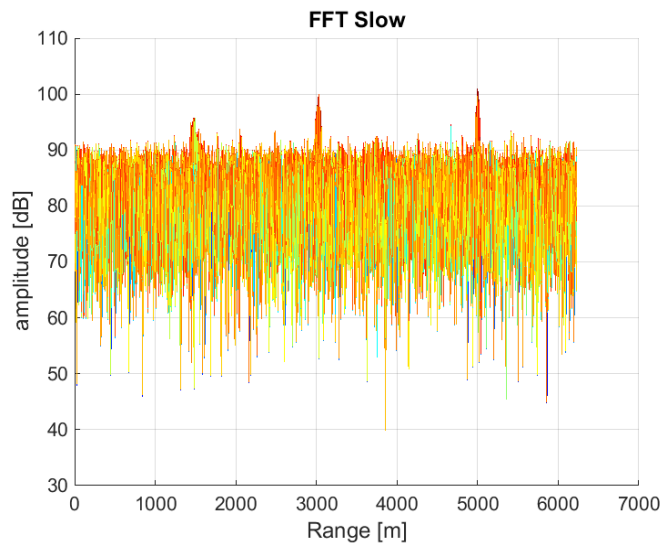

Fig. 11: 2D spectrum of the signal with three weak targets (Power-Range)

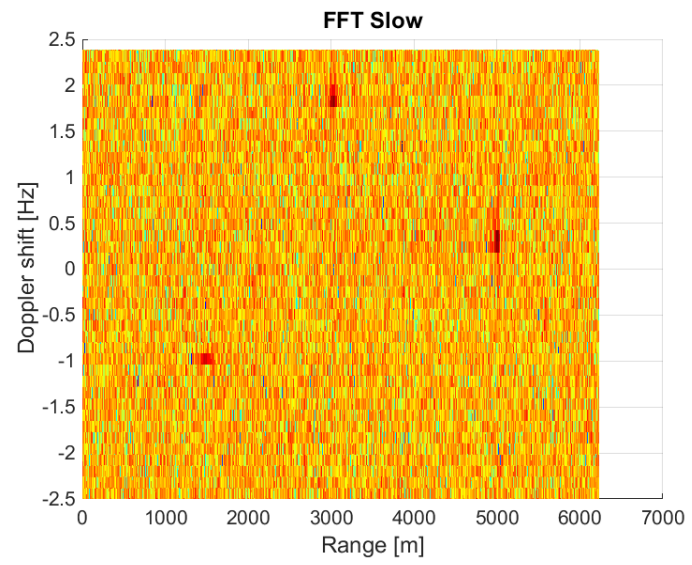

Fig. 12: 2D spectrum of the signal with three weak targets (Doppler shift-Range)

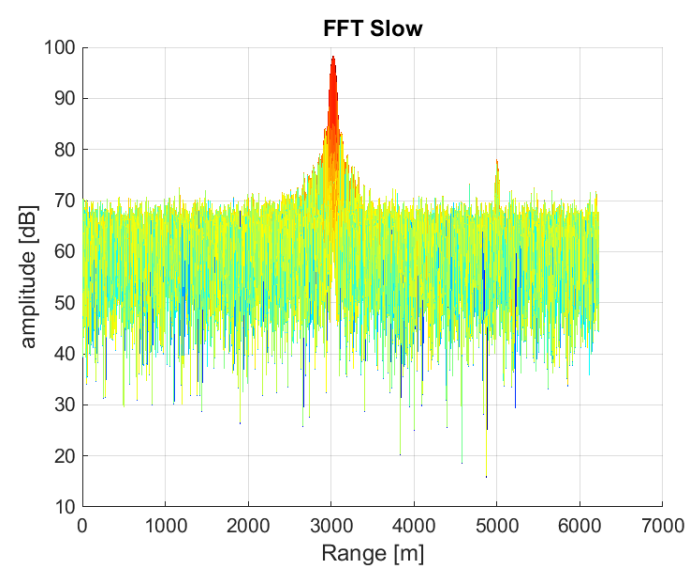

Fig. 13: 2D spectrum of the signal with one strong and one weak target (Power-Range)

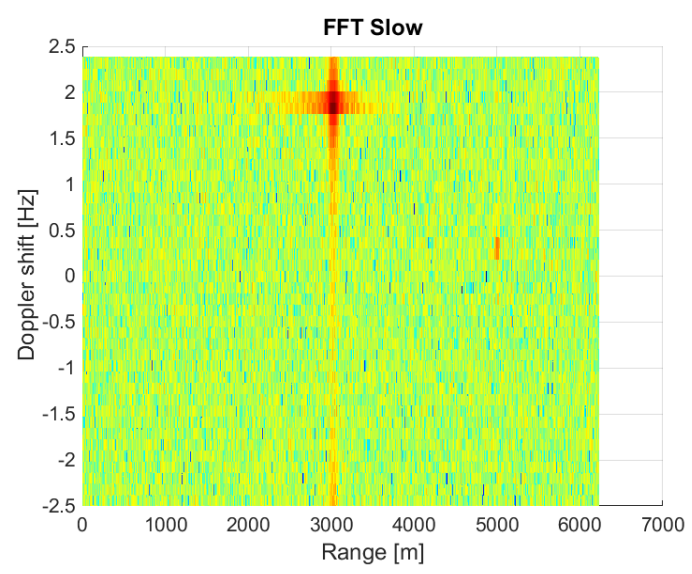

Fig. 14: 2D spectrum of the signal with one strong and one weak target (Doppler shift-Range)

Matrix S2D after thresholding is shown in Fig. 16. This matrix is in the next step used for the calculation of the range profile. This profile is a vector. Each cell in the range vector is number of all points (in Doppler shifts) higher than the threshold value (blue line in Fig. 17). For the noise removal the moving average filter of the fifth order was used. It is shown in Fig. 17 by the red curve. Green line is threshold value. If the number of Doppler shifts (after thresholding and filtration) is one or bigger, the target on this position is used in next signal processing. Number of the targets is calculated in the next step. This calculation is realized by the edge 
detector. Number of the rising edges is similar to the number of the targets. The filter of the fifth order was used for filtration and border samples are removed from the processing (two first samples and two last samples - they were not filtered). Position of the maximum (between the two rising edges after thresholding) in the range profile leads to the real position of the target estimation. Doppler shift is calculated for thus derived distance.

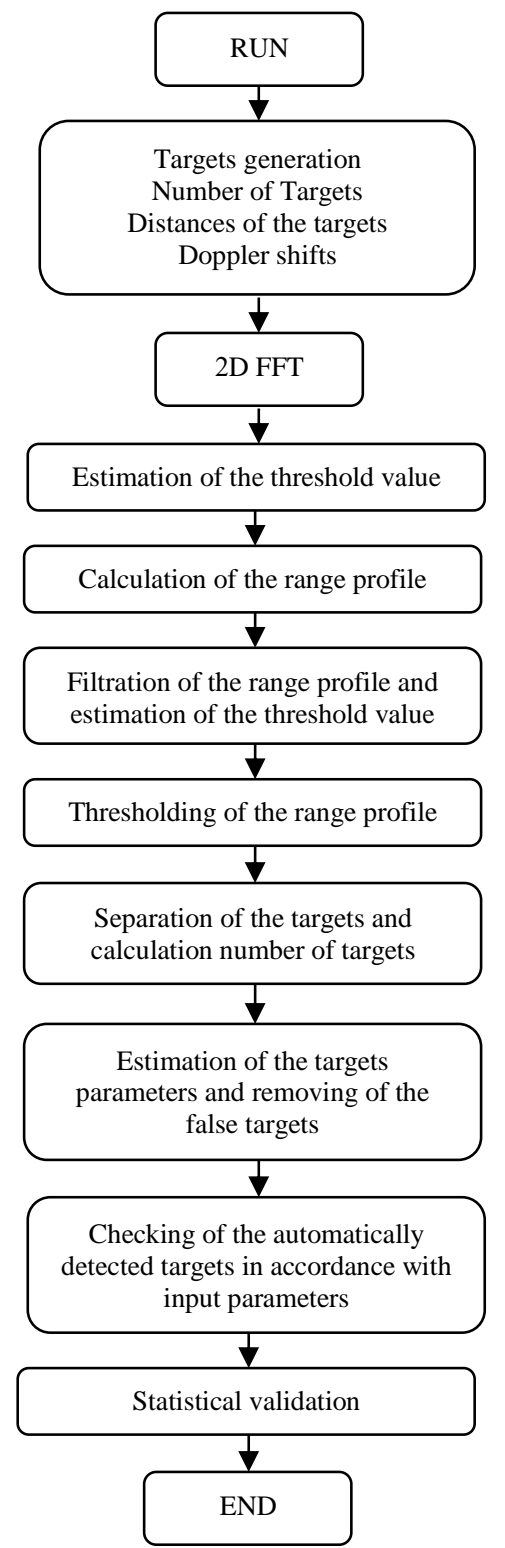

Fig. 15: Algorithm for the signal processing with implemented points for the calculation of the algorithm efficiency

The Doppler value is calculated as Doppler shift of the maximal peak in the spectrum in the monitored distance. This Doppler shift is used for the false alert elimination (if the Doppler shifts of two targets are close each other). The target with smaller maximal value is removed from the processing as the false alert.

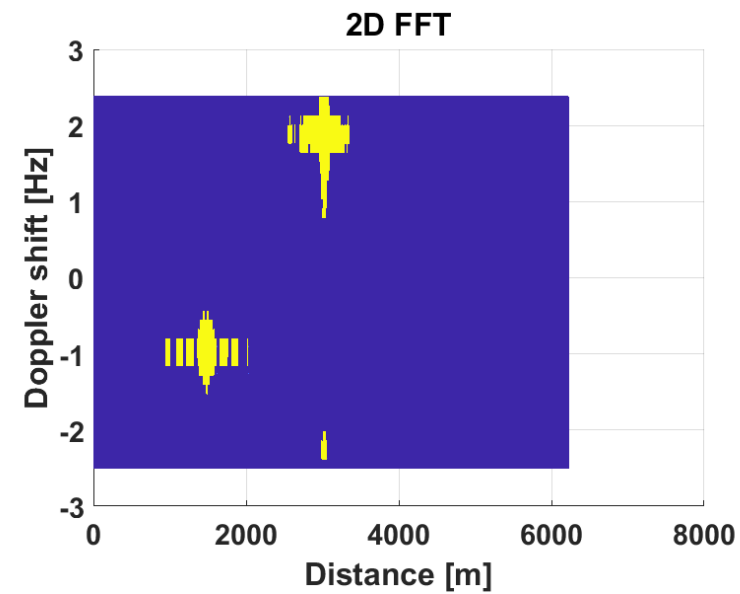

Fig. 16: Spectrum after thresholding with two targets

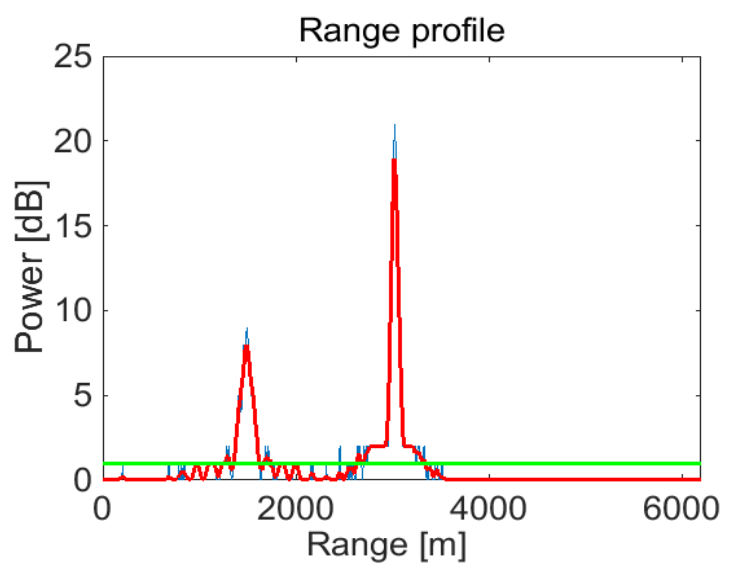

Fig. 17: Range profile of the signal with two strong targets

\section{Results from the experiment and discussion}

Results from the experiment are in the Table 1. 7 types combination of the simulated targets were selected for the test. The first signal is noise without targets (Fig. 7 and Fig. 8). The second signal contains one weak target. The third signal contains two weak targets. The fourth signal has three weak targets (Fig. 11 and Fig. 12). The fifth signal contains one strong target. The sixth signal has two strong targets (Fig. 9 and Fig. 10). The seventh signal contains one strong target and one weak target (Fig. 13 and Fig. 14).

All types of the signals were generated 100 times. After processing the results were compared with input parameters. If the number of the targets was estimated wrongly, other parameters of this signal (distances of the targets and Doppler shifts of the targets) were not checked and the result was evaluated as wrongly processed. If the distances of the targets were not estimated in the input intervals, Doppler shifts were evaluated as wrongly processed.

Test of the signals without targets shows good results. Only three measurements were processed wrongly. Unsuccessful cases were caused by the unfavorable combinations of the noise peaks in the spectrum. Results for the distances and Doppler shifts were not calculated (there is no target for analysis of the signal).

The second part describes analysis of the signal with weak targets. Weak targets were selected with 
signal noise ratio in interval from 5 to $10 \mathrm{~dB}$. Failures of the algorithm for 2 and 3 weak targets were caused by target with signal noise ratio $5 \mathrm{~dB}$ at distance $1500 \mathrm{~m}$ (Fig. 11). It is possible to see that the algorithm can be used for the target detection of minimal signal noise ratio approx. $9 \mathrm{~dB}$ (simulated target at distance $3000 \mathrm{~m}$ - used in the all three combinations with weak targets).

The third part is the analysis of the signal with strong targets. These signals have problem with the next replications of the one target (with the same Doppler shift but of different distances). This problem is caused by side lobes in the spectrum. It is shown in Fig. 9 and Fig. 10. For these side lobes removing we used condition in algorithm for removing of the targets in a row with the same Doppler shifts (it is probably caused more by the presence of the side lobe than by presence of two targets with the same Doppler shifts).

The last part is combination of a strong and a weak target. We can see that the algorithm can be used for the target detection in this combination of the targets. But if the distance between targets is very small, weak target can be lost.

Table 1: Results from the test of the algorithm

\begin{tabular}{cccc}
\hline Tested targets & Correctly detected targets (\%) & Correctly estimated distance (\%) & Correctly estimated Doppler shift (\%) \\
\hline 0 targets & 97 & --- & -- \\
1 weak & 97 & 97 & 97 \\
2 weak & 86 & 78 & 77 \\
3 weak & 85 & 79 & 78 \\
1 strong & 99 & 98 & 98 \\
2 strong & 99 & 97 & 97 \\
1 weak + 1 strong & 98 & 96 & 96 \\
\hline
\end{tabular}

\section{Conclusion}

Algorithms for automatic detection of the targets are important for the driving of the autonomous systems for protection against collision (ships, airplanes, bots in the factory and others). In ideal case, combination of the visual systems, ultrasound systems and radio systems is to be used. Visual systems can have problem during the fog, ultra sound systems have problem with vibration of devices and electromagnetic systems can fail due to electromagnetic interference (in dependence on frequency: Wi-Fi networks, radio links, communication systems and other).

Designed algorithm was tested on four types groups of the simulated signals (noise, weak targets, strong targets, combined targets). All tested variants were highly successful. Only signals with very low signal noise ratio were problematic. However in the real processing only signals with signal noise ratio $10 \mathrm{~dB}$ are used for our analysis.

From the results we can conclude that the proposed algorithm can be used for target detection with signal noise ratio higher than $9 \mathrm{~dB}$. If this condition is fulfilled, algorithm has a high chance to succeed.

Problem of this algorithm is only detection of the targets with the same Doppler shift in one moment. However separation of these two targets is very often problematic even for direct manual analysis.
Our plane for future is the modification of the described algorithm for real (measured) data on one hand and modification of the algorithm for description of the volume targets (e.g. rain cells) on the other hand.

\section{Acknowledgment}

This paper was supported by internal grant University of Pardubice SGS FEI_2016_22 and by the grant GAČR 15-24688S.

\section{References}

Babur G, Krasnov OA, Yarovoy A, and Aubry P (2013). Nearly orthogonal waveforms for MIMO FMCW radar. IEEE Transactions on Aerospace and Electronic Systems, 49(3): 1426-1437.

Mandlik M and Brazda V (2015). FMICW radar simulator. In the $25^{\text {th }}$ International Conference on Radioelektronika, IEEE: 317 320. https://doi.org/10.1109/RADIOELEK.2015.7129054

Rejfek L, Bezousek P, Fiser O, and Brazda V (2014). FMICW radar simulator at the frequency $35.4 \mathrm{GHz}$. In the $24^{\text {th }}$ International Conference on Radioelektronika, IEEE, Bratislava, Slovakia: 14. https://doi.org/10.1109/Radioelek.2014.6828452

Rejfek L, Mošna Z, Urbář J, and Koucká Knížová P (2016). System for automatic detection and analysis of targets in FMICW radar signal. Journal of Electrical Engineering, 67(1): 36-41.

Řezníček M, Bezoušek P, and Zálabský T (2016). Amti filter design for radar with variable pulse repetition period. Journal of Electrical Engineering, 67(2): 131-136. 